\title{
Rhythmisches zu der Kunstprosa des Konstantinos Manasses.
}

Da die Edition der Blüten byzantinischer Kunstprosa an der Tagesordnung zu sein scheint, so ist es Zeit, auf ein für die Textkritik entscheidendes Moment aufmerksam zu machen, das bisher völlig unberücksichtigt geblieben ist. Über die rhythmische Behandlung dieser Prosa ist aufser dem Meyerschen Satzschlufsgesetz ${ }^{1}$ ) nichts bekannt; ich stelle hier kurz zusammen, was ich aus den bisher veröffentlichten Stücken des Konstantinos Manasses (Ende des XII saec. $)^{2}$ ) von diesem Standpunkt aus nachzutragen fand.

1) Im Ausgang der Satzglieder mufs die Zahl der zwischen den letzten beiden Hochtönen stehenden Silben eine gerade sein; d. h. ein Zwischenraum von $0,1,3,5$ und 7 Silben ist ausgeschlossen. Wie weit der Begriff „Satzglied“ im Sinne dieses Gesetzes zu fassen ist, kann ich noch nicht in einer unzweideutigen Definition ausdrücken. Über das, was unsere Interpunktion anzeigt, geht er in der Regel weit hinaus; unter Umständen unberücksichtigt bleibt nur

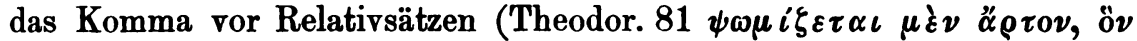

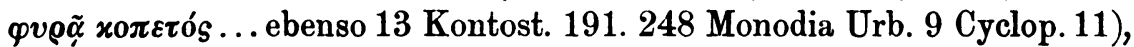

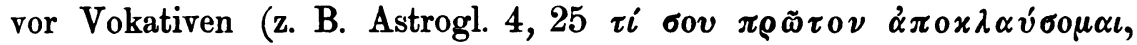
$\mu 0 v \sigma \iota x \dot{\sigma} \tau \alpha \tau \varepsilon$;) und zwischen gleichartigen kurzen asyndetischen Sätzen oder Satzteilen (Cyclop. $50 \delta \alpha \sigma \grave{v} S \tau \grave{\eta} \nu$ xó $\mu \eta \nu, \pi 0 \lambda \dot{v} S \tau \grave{\alpha} S$

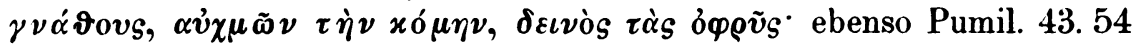

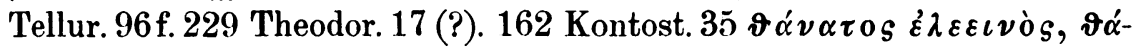

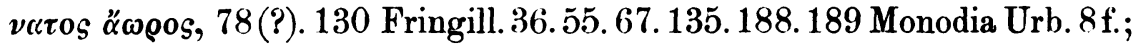
Theodor. 7, 79. $84 \tau \dot{\eta} \nu \pi \varepsilon \rho \iota \sigma \tau \varepsilon \rho \dot{\alpha} \nu \tau \grave{\eta} \nu x \alpha \lambda \lambda \iota \pi \tau \varepsilon \dot{\varepsilon} v \gamma \alpha, \tau \dot{\eta} \nu \dot{\alpha} \varrho \gamma v \rho \circ \pi \tau \varepsilon^{\prime}-$

1) Wilhelm Meyer (aus Speyer), Der accentuierte Satzschlufs, Göttingen 1891 und C. Litzika, Das Meyersche Satzschlufsgesetz, München 1898.

2) Abkürzungen: Theodor. = Monodie auf die Theodora; Kontost. = Consolatio an den Johannes Kontostephanos (beides ed. E. Kurtz, Viz. Vremenn. VII $621 \mathrm{ff}$.); Astrogl. = Monodie auf den Astroglenos (ed. K. Horna); Pumil. Tellur. Cyclop. Fringill. = Beschreibung des Zwergen, des Erdbildes, des Cyklopenbildes, des Vogelfangs; Monodia Urb. = Monodie auf den Unbekannten (die letzten 5 Stücke ed. L. Sternbach); zur Bibliographie vgl. K. Krumbacher, $\mathrm{GBL}^{2} 380,4$ und $\mathrm{BZ}$ oben 209 und unten $580 \mathrm{f}$. $659 \mathrm{f}$. 
$\varrho v \gamma \alpha, \tau \grave{\eta} \nu \chi\llcorner o \nu \omega ́ \delta \eta, \tau \grave{\eta} \nu \pi \alpha ́ \lambda \lambda \varepsilon v \varkappa o \nu$ und so öfters). Im allgemeinen aber wird jeder in sich geschlossenen Wortkomplex, der gegen den folgenden Komplex abgeschlossen werden kann, ohne dafs dieser. letztere zu kurz gerät, als Satzglied im Sinne der oben genannten Regel behandelt; so kommen deren durchschnittlich zwei auf die Druckzeile. Einen „Hochton“ hat jedes accentuierte Wort, mit Ausnahme von Partikeln, Konjunktionen, Präpositionen, Negationen etc.

Also Manasses begnügt sich nicht mit der Wahrung des Meyerschen Gesetzes, sondern er meidet erstens auch das Intervall von 3 Silben, was L. Havet (Revue critique XXXII 208) als dem Sinn jenes Gesetzes entsprechend erkannt, aber noch niemand für irgend einen Autor nachgewiesen hat (rgl. Meyer S. 11), zweitens aber auch das Intervall von 5 und 7 Silben, ein Moment, das überhaupt noch nicht erwähnt ist. Die Ursache davon ist nicht in der grofsen Entfernung der letzten Hochtöne zu sehen, wie die Häufigkeit (59 Beispiele) 6 silbiger

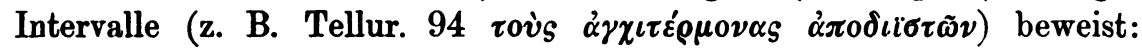
Theodor. 7. 11 f. 74. 78. 128. 156. 180 Kontost. 20. 25. 34. 43. 52. 54 . 66. 91. 113. 130. 134. 167. 206. 211. 242. 265. 287. 299. 300. 304. 307. 319 Astrogl. 3, 24. 4, 20. 5, 3. 13. 6, 30. 8, 20. 9, 3 Pumil. 19. 40 Tellur. 58.94.178 Cyclop. 23. 53. 67 Fringill. 4. 36. 62.71 (statt $\delta$ ' lies $\delta \xi$ ). 90.92. 95. 116. 132. 135. 156. 179 Monodia Urb. 4. 14; vielmehr kommt bei einer ungeraden Zahl unbetonter Silben durch die unbewufst alternierende Verteilung der Nebentöne ( $v \backsim v \sim v \sim v-)$ ein wenn auch schwacher Accent auf die vorletzte Silbe, was den Rhythmus retardiert und so gegen das Grundgesetz der prosaischen Accenttechnik verstölst. ${ }^{1}$ )

1) Am nächsten steht dem Manasses in dieser Beziehung das 'Eyxíuıov xvvós des dem Manasses gleichzeitigen Professors der Rhetorik Nikephoros Basilakes (ed. E. Miller, Mélanges orientaux, Paris 1883, p. 255 ff., vgl. GBL ${ }^{2} 475,3$ ). In diesem Stück ist das oben genannte Accentgesetz nur einmal durchbrochen:

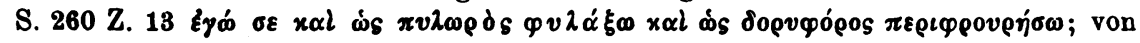
Druckfehlern abgesehen, bleibt nur eine Stelle zu korrigieren: S. 261 Z. 8 von

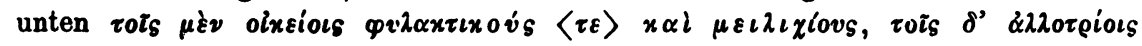

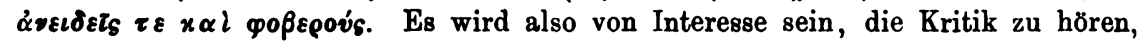
die Basilakes in der Vorrede zu seinen Werken (ed. E. Miller, Annuaire de l'assoc. pour l'encour. des études gr. VII [1873] $133 \mathrm{ff}$.) den Rhythmen seiner eigenen Kunst-

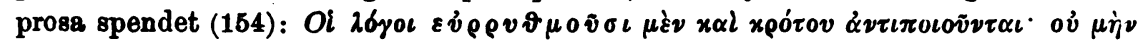

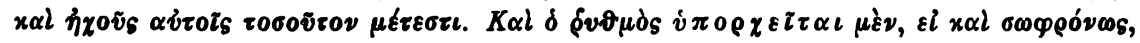

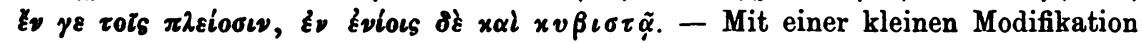
(8. darüber S. 510 Anm. 2) ist jenes Accentgesetz streng durchgeführt in den Reden des Nikephoros Chrysoberges (ed. M. Treu, Programm des FriedrichsGymn. Breslau 1892), ebenfalls eines Zeitgenossen des Manasses. Unter den übrigen erwähne ich hier nur den Michael von Thessalonike (ed. W. Regel, Fontes rerum byzantinarum I 1, 131-182), der das Accentgesetz nicht ganz so streng befolgt und gegen 6 silbige Intervalle eine grofse Abneigung zeigt. 
Bei der Betrachtung der Ausnahmen bitte ich zu bedenken, dafs sie sich gegen die Masse von fast 2500 regelmälsigen Schlüssen abheben. Wörtliche Bibel- oder Homerzitate sind natürlich nicht berücksichtigt. Zusammenstöfse von Hochtönen am Schlufs eines Satz-

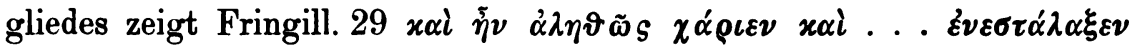

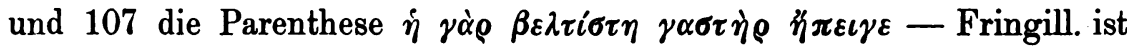
überhaupt bezüglich der Schlufsaccente am nachlässigsten behandelt

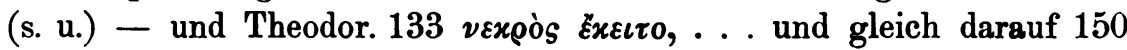

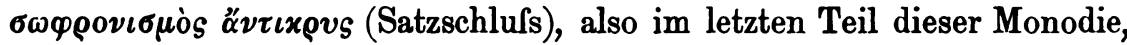
der auch aufserhalb des Schlusses besonders häufig Zusammenstofs von Hochtönen zeigt (s. u. unter 2). Intervalle von 1 Silbe finden sich

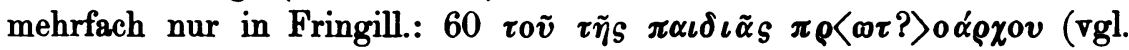

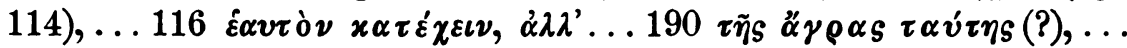

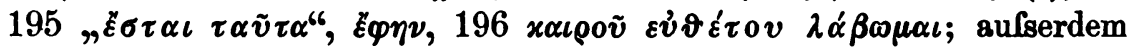

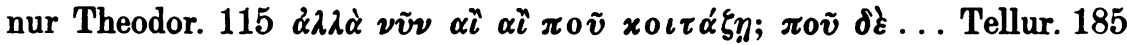

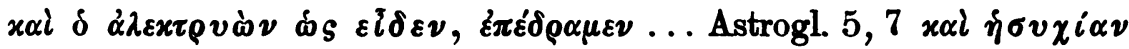

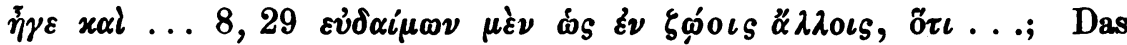
übrige gehört den Schreibern oder Herausgebern: Kontost. $254 \Pi \alpha \tilde{v} \lambda_{0}$

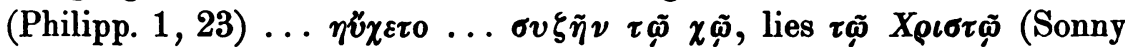
BZ X 659); dann betreffend die Accentuation der Enklitika ${ }^{1}$ ): Theodor.

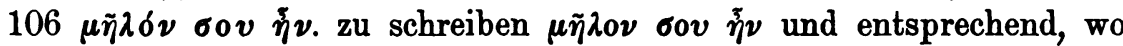

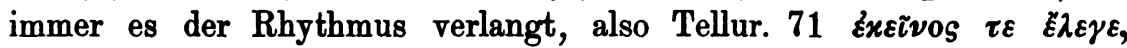

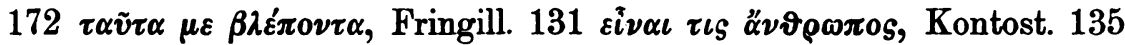

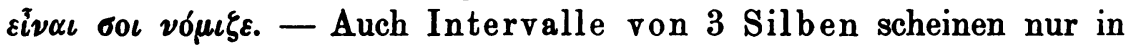
Fringill. zugelassen zu sein: $33 x \alpha \tau \alpha \tau \rho \dot{v} \gamma \eta s \dot{v} \psi \eta \lambda \delta$ ó $44 \sigma \tau 0 \iota x \eta \delta \dot{o} \nu \delta \xi x \alpha \tau \varepsilon \tau \alpha^{\prime} \sigma \sigma 0 \nu \tau 0^{\circ} \ldots 46 \varepsilon^{\prime} x \varepsilon \ell \nu \alpha s \pi \alpha \rho \varepsilon \pi v \dot{v} x \alpha \xi \varepsilon \nu, \ldots 65(?) \ldots$

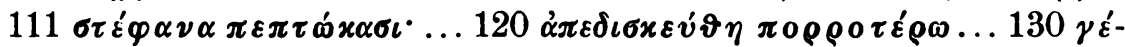

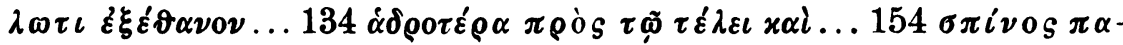

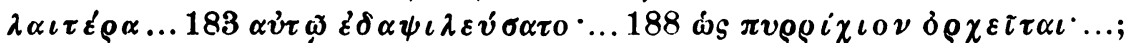
in den übrigen Schriften fand ich nur Theodor. 7 ßo $\tilde{\alpha} \gamma \dot{\alpha} \varrho \dot{\alpha} \nu v \mu \varepsilon \dot{\varepsilon} \alpha \iota \alpha$

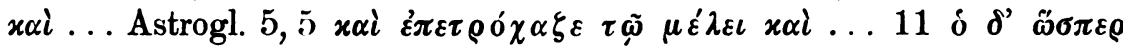

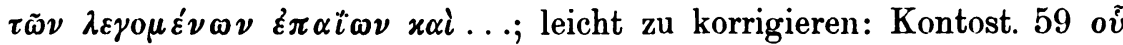

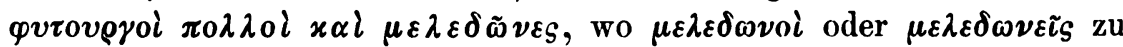
lesen sein wird; denn warum sollte sich Manasses einer unklassischen Form zulieb seinen Rhythmus verdorben haben? Kontost. 20 oivov...,

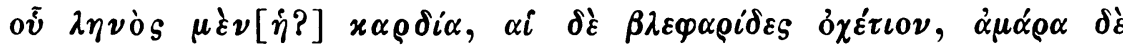

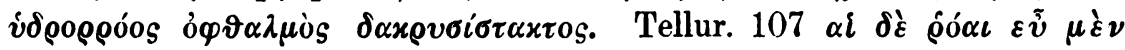

1) Wie geschmeidig sich die Enklitika bei den Byzantinern den jeweiligen Forderungen des Rhythmus fügt, habe ich in einem Exkurs zu meiner Studie „Der byzantinische $\mathrm{Zwölfsilber"} \mathrm{zu} \mathrm{zeigen} \mathrm{versucht;} \mathrm{diese} \mathrm{mufste} \mathrm{aber} \mathrm{für} \mathrm{das}$ niichste Heft der $\mathrm{BZ}$ zurückgestellt werden. 


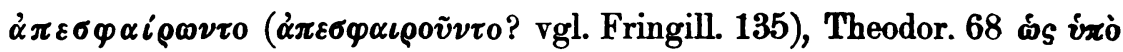

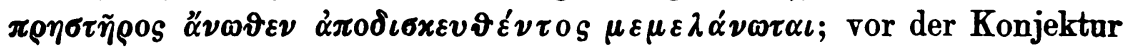
$\mu \varepsilon \mu \varepsilon \dot{\lambda} \alpha \nu \tau \alpha \iota$ bewahrt uns die Hs C, die vor $\mu \varepsilon \mu \varepsilon \lambda \alpha^{\prime} \nu \omega \tau \alpha \iota$ einschiebt: $\tau \dot{\eta} \nu$ $\pi \varepsilon \rho \iota \beta 0 \lambda \dot{\eta} \nu$. Es war unmethodisch, diese Lesart nicht in den Text zu setzen; denn derartige Glosseme sind dieser Art von Überlieferung fremd, der Ausfall von Worten aber nicht. Kontost. $105 \tau \dot{\eta} \nu$ ov́ $\tau \omega x \alpha \lambda \dot{\eta} \nu, \tau \dot{\eta} \nu$

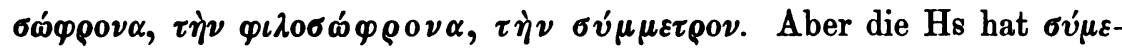
$\tau \varrho \circ \nu$ (so!) nur über der Linie, im Text steht $x \alpha \lambda \eta \nu \nu$. Es ist beides falsch, das Richtige kaum zu finden. Die Behandlung der Enklitika ist zu

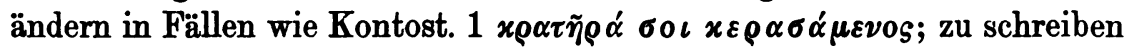

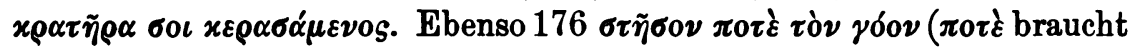

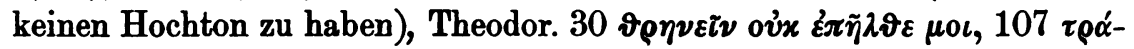

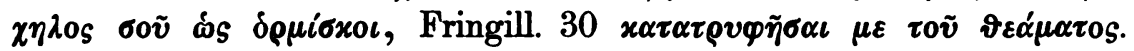

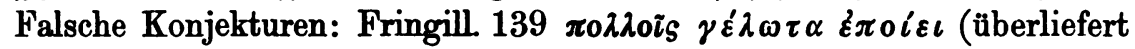

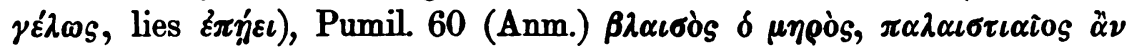

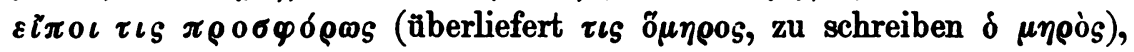

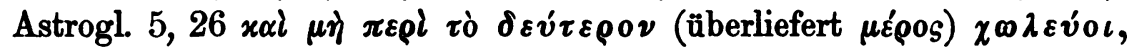

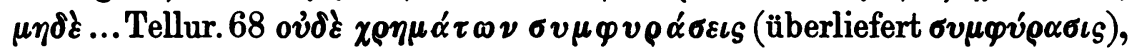
sowie Cyclop. 44 (Anm.) 73. 79 die freien Ergänzungen des Herausgebers. Jetzt wissen wir auch, warum Cyclop. 28 (Anm.) die Spuren der Hs auf

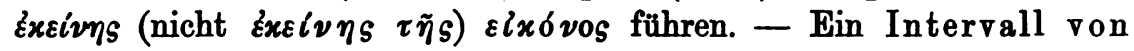
5 Silben scheint zweimal sicher bezeugt zu sein: Theodor. $112 \dot{\alpha} \rho-$

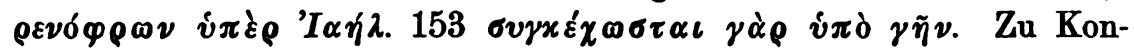

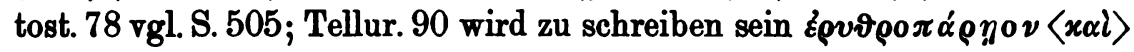

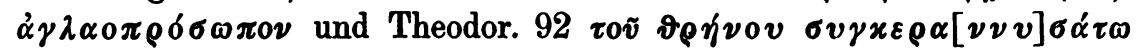
(oder $\sigma v \gamma x \varepsilon \rho \alpha \nu \nu v[\sigma \alpha] \tau \omega)$. Zur Behandlung der Enklitika: Theodor. 105

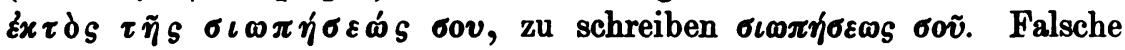

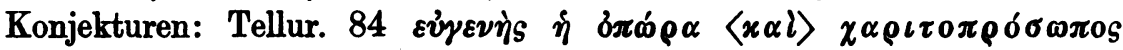
(Hercher), mit Recht von dem letzten Herausgeber zurtakgewiesen; nur

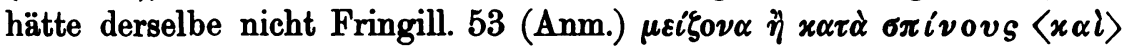
$\beta \alpha \rho v \varphi \omega \nu \delta$ $\varepsilon \rho \alpha$ denselben Fehler machen sollen. Astrogl. 4, $25 \pi \varepsilon v-$

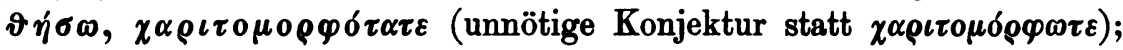
Tellur. $21 \dot{\alpha} \pi \alpha \gamma o \rho \varepsilon v ́ \sigma \varepsilon \iota \varepsilon$ (überliefert - $\varepsilon l$, vgl. Sternbach zu Fringill. 185)

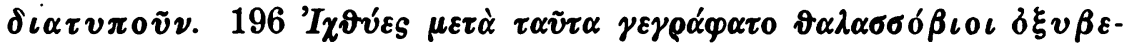

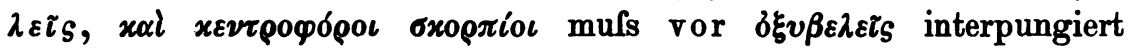
werden. - Ein Intervall von 7 (oder mehr) Silben fand ich nirgends;

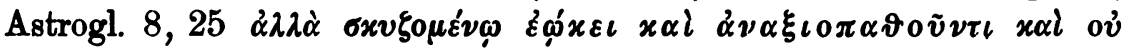
ตย́ durch eine Art Hendiadyoin mit dem folgenden verbunden ist.

2) Abneigung gegen das Zusammenstofsen von Hochtönen im Innern eines Satzgliedes (vgl. Meyer S. 1). Damit ist der wichtigste 
Teil des Satzgliedschlufsgesetzes, wodurch das Brechen des Rhythmus verwehrt wird, auf das Innere ausgedehnt; es ist nur zu verwundern, dafs es damit bei Manasses nicht zu einer Regel gekommen ist. Auch die Abneigung zeigt sich in Fringill. Tellur. Cyclop. nicht, wir können vielleicht sagen: noch nicht; in den übrigen Stücken aber ist sie deutlich. Wenn man von Redensarten wie $\varepsilon \tilde{\omega}$ $\lambda \dot{\varepsilon} \gamma \varepsilon \iota \nu$ Pumil. 17 Astrogl. 3, 22,

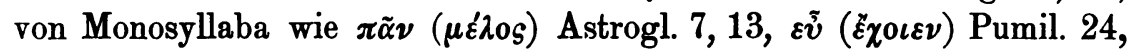
$\tau i(\varepsilon \tilde{\tau} \iota)$ Kontost. 243, von Verbindungen sonst hochtonloser Monosyllaba

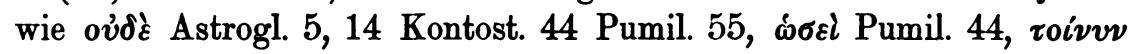

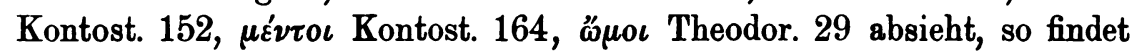
sich in den 25 Zeilen der Monodia Urb. und den ersten 157 Zeilen von Theodor. (1 zwischen É $\gamma \grave{\omega}$ und $\pi \alpha ́ \lambda \iota \nu$ zu interpungieren) keine Ausnahme, in Kontost. Zeile 1-103 und 136-324 (Ende) zusammen 6 Ausnahmen (1.29. 74.203. 234. 252), in den 91 Zeilen von Pumil. 1 Aus. nahme (35), in den 189 Zeilen von Astrogl. 3 Ausnahmen (6, 24.

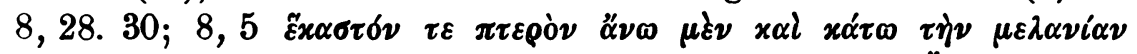

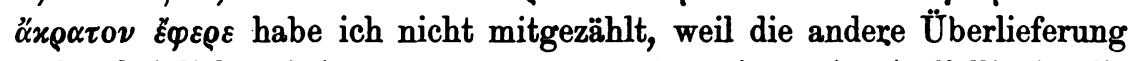

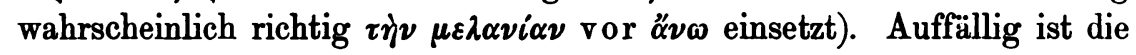
Häufigkeit der ZusammenstöIse an den oben ausgelassenen Stellen von Theodor. und Kontost.; dort finden sich in den letzten 30 Zeilen 6 Ausnahmen (158. 160. 164. 174. 175. 181), hier in 32 Zeilen 6 Ausnahmen (104. 111. 114. 120. 125. 135). Aber die Deutlichkeit, mit der die Abneigung in Pumil. und Astrogl. zu Tage tritt, berechtigt wohl dazu, die Spuren derselben in den übrigen Stücken nicht dem Zufall zuzuschreiben. ${ }^{1}$ )

3) Vorliebe für Hochton auf der drittletzten und Abneigung gegen Hochton auf der vorletzten Silbe am Satzschlufs. Die Behandlung der auf den letzten Hochton folgenden Silben lüfst sich zwar nicht in ein für zwölf Jahrhunderte geltendes Gesetz fassen; doch ist sie bei der Untersuchung über einzelne Autoren und Zeiten ein wichtiges Moment. Die Vorliebe für proparoxytonischen Schlufs, die sich bei Sophronius in der von E. Bouvy (Poètes et mélodes, Nîmes 1883, p. 201 ff.) nachgewiesenen regelmälsigen Verwendung des „Doppeldaktylus“ äufsert, durchzieht, mehr oder minder stark, die

1) In dem 'Eyxónıov xvvós des Nikephoros Basilakes (113 Zeilen) finden sich nur zwei Zusammenstöfse von Hochtönen: S. 259 Z. 8 (zwischen Satzgliedern?) und S. 261 Z. 13 von unten; schon deshalb ist S. $258 \mathrm{Z} .9$ von unten nicht $\pi \tau \eta$ $\nu \tilde{\omega} \nu\left\langle\varepsilon \tilde{l}^{\dagger}\right\rangle \lambda \alpha \iota_{\xi}$ zu ergänzen, sondern $\left\langle\dot{\alpha} \gamma \dot{\varepsilon}^{\prime}\right\rangle \lambda \alpha \iota$ s. Noch strenger sind in dieser Beziehung die Reden des Nikephoros Chrysoberges gehalten; hier finden sich in 1150 Zeilen nur 4 sichere Ausnahmen: 1, 24. 4, 33. 10. 36 (Psalm 2, 9). 16, 31;

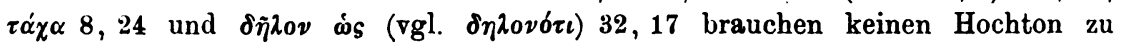
haben; korrupt scheint 28,28 ( $(\dot{v} \tau \varepsilon \tilde{v} \vartheta \varepsilon v$ statt $\xi \nu \vartheta \varepsilon v$ ?); Konjekturen 11, 2. 33, 30. 
gesamte byzantinische Kunstprosa; als nächstberechtigt erscheint der endbetonte Schlufs, offenbar weil auch bei proparoxytonischen ein Nebenton auf die letzte Silbe fällt; der Hochton auf der vorletzten hingegen war ganz andersartig und wird wohl aus diesem Grund von vielen Autoren gemieden. ${ }^{1}$ ) Bei Manasses zeigt sich dies hauptsächlich vor grölseren Sinnespausen: unter den von den Herausgebern im Druck gekennzeichneten Absätzen haben 31 den letzten Hochton auf der drittletzten, 13 auf der letzten und nur 3 auf der vorletzten Silbe (Kontost. 289 Tellur 79 Astrogl. 8, 2i); für die im Text durch einen Punkt abgeschlossenen Perioden bei Kontost. sind die entsprechenden Zahlen 32,18,6. ${ }^{2}$ )

4) Streben nach Isosyllabismus und Isotonismus in parallelen Sätzen und Satzteilen. ${ }^{3}$ ) Beispiele dafür finden sich nur in Pumil. (hier ohne Isotonismus) und Astrogl. Ich schreibe die deutlichsten aus.

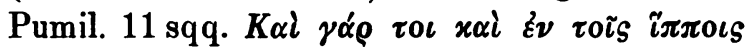

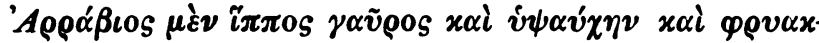

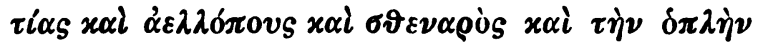

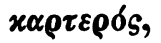

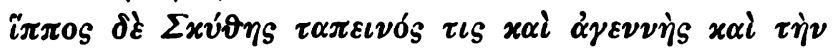

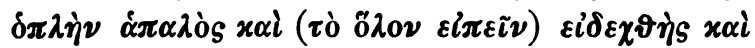
$\chi \alpha \mu \alpha i \xi \eta \lambda \sigma^{\circ}$

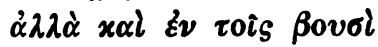

1) Oder sollte hier, wie z. B. in der antiken Rhetorik, die Abneigung gegen den der Poesie (Acht-, Zwolf-, Fünfzehnsilber) charakteristischen Schlufs mitspielen?

2) In dem 'Eyxóntov xvvós des Nikephoros Basilakes: 21, 4, 1; bei Michael von Thessalonike ist hochbetonte Pänultima von allen Satzschlüssen geradezu ausgeschlossen, wăhrend endbetonte Schlüsse kaum beschränt erscheinen (für die Absătze des Textes ergeben sich die Zahlen 24, 20, 0); Nikephoros Chrysoberges geht noch weiter: er läfst hochbetonte Pänultima auch im Satzglied achlusse (nach der unter 1 gegebenen Definition) nur ausnahmsweise zu, und seltsamer Weise fallt der grofste Teil dieser Ausnahmen mit denen gegen das unter 1 genannte Gesetz zusammen. Ich gebe hier die Belege für die gröfseren Inter-

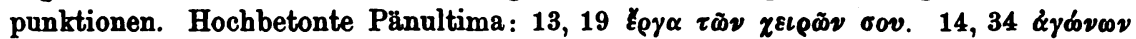

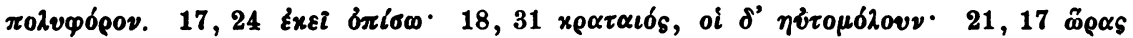

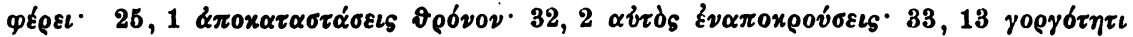
$\pi \lambda \alpha \tau \dot{v} \omega \nu$. Ungerade Zahl unbetonter Silben zwischen den letzten Hochtönen: aufser

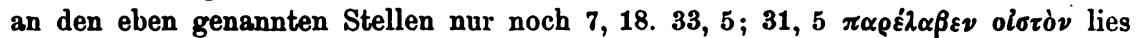
bǐoróv. Die kleineren Sinnespausen werden nur wenig freier behandelt. - Auch bei Manasses zeigen die unregelmäfsigen Schlüsse (S. 507) auffällig oft betonte Pünultima.

3) Auch dies ist eine Eigentümlichkeit des Nikephoros Basilakes, wenn

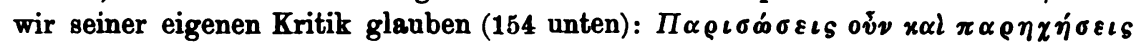

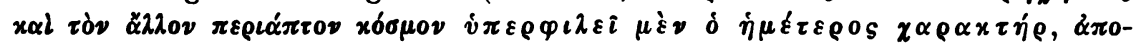

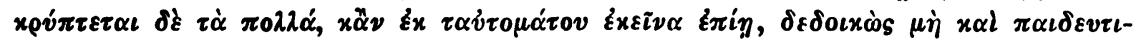

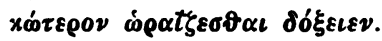




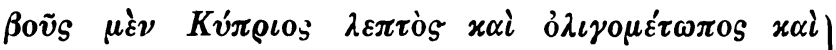

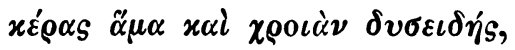

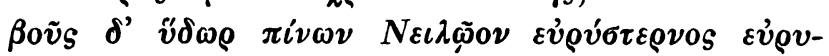

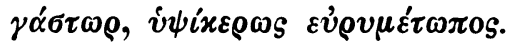

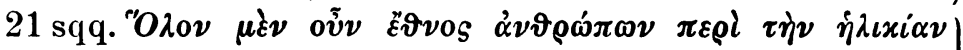

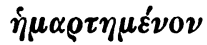

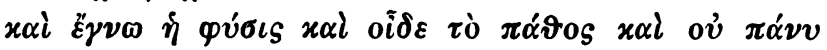

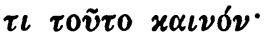

$\dddot{\alpha} \nu \delta \varepsilon \dot{\varepsilon} \tau \iota \nu \iota$

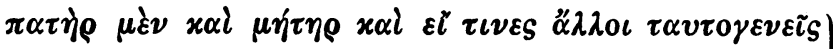

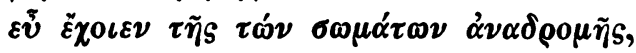

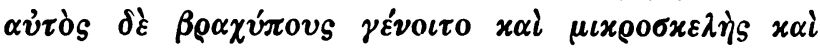

(

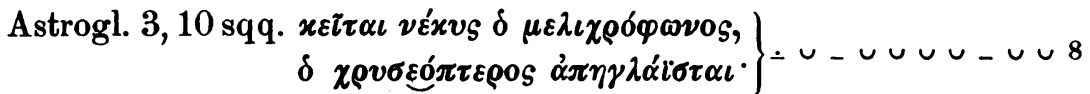

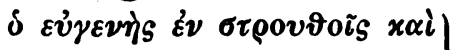

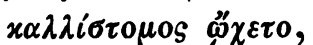

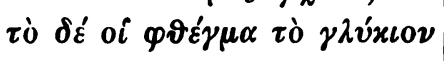

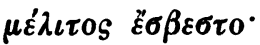

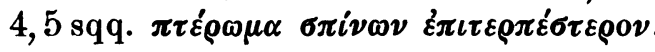

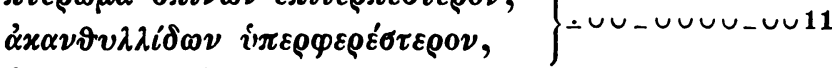

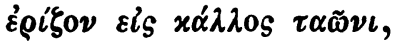

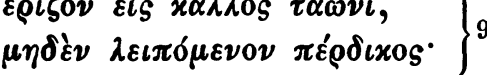

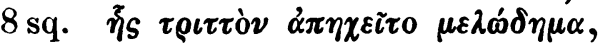

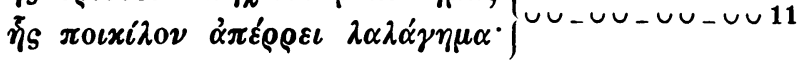

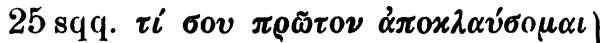

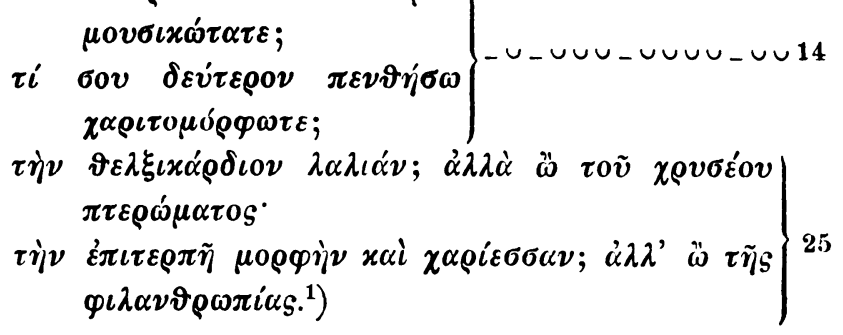

1) Nah verwandt hiermit ist eine Stelle aus des Euthymios Monodie auf

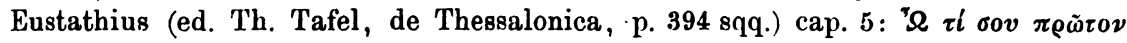

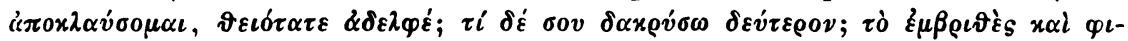

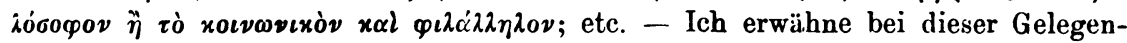
heit noch eine andere viel auffälligere Parallele, die man vielleicht besser Plagiat nennt: Manasses Tellur. 119-143 stimmt meist wörtlich ïberein mit dem Anfang der dem 'I'heodoros Prodromos zugeschriebenen $\Sigma \chi \xi \delta \eta \mu v \sigma_{s}$ (ed. Boissonade, Anecd. Graec. I 429 sqq.).

Byzant. Zeitschrift XI 3 n. 4. 


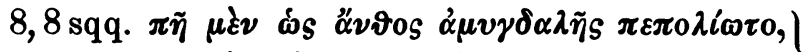

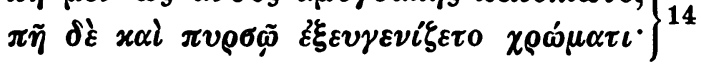

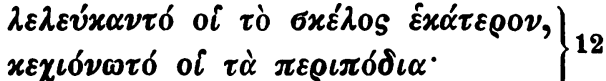

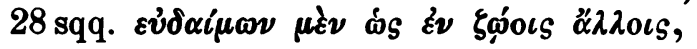

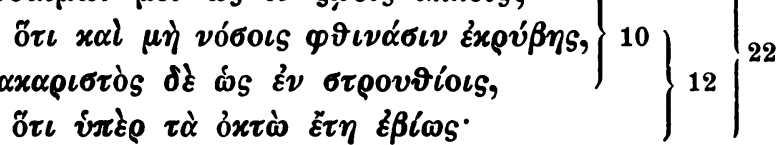

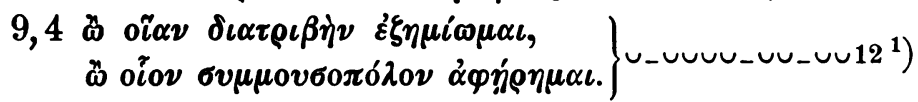

Es wird mir vielleicht später einmal möglich sein, die hier besprochenen rhythmischen Regeln in ihrer Entwicklung darzustellen; denn dafs sie nicht Alleinbesitz des Konstantinos Manasses sind, vielmehr zum mindesten einer ganzen Gruppe von Rhetoren des ausgehenden XII saec. gehören, haben die Anmerkungen, deren Ausdehnung sich leicht hätte verdoppeln lassen, wohl genügend bewiesen. Doch hoffe ich auch jetzt schon der niederen wie der höheren Textkritik ein bedeutend feineres Instrument an die Hand gegeben zu haben, als es die bisher bekannten Thatsachen lieferten; möge es den vielen unedierten Schriften, die noch im Schofs der Bibliotheken schlummern, oder sich bereits aus den Schreibtischen der Gelehrten nach dem Licht der Welt heraussehnen, zu Gute kommen! Wenn ich aber durch den Hinweis auf die bedeutenden Feinheiten dieser Kunstprosa aufserdem noch erreicht habe, dals man aufhört, in den byzantinischen Rhetoren nur eine Herde von gewissenlosen Schmeichlern und aufgeblasenen Tröpfen zu sehen, so wird dies der objektiven Beurteilung jener ganzen Epoche gewifs nicht schaden.

München.

\section{Paul Maas.}

1) Ich bin vom Text abgewichen: Pumil. $\left.\left.14 x \alpha i \xi_{\nu}\right] x \alpha^{2} \|{ }_{16} \delta^{\prime}\right] \delta^{\prime} \xi \|$ Astrogl. 4, 27

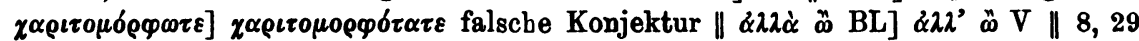

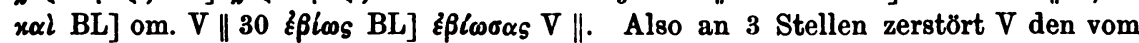
Rhetor zweifellos erstrebten Isosyllabismus; und in der That bietet diese Hs, obwohl auf sie die Ausgabe gebaut ist, die schlechtere Uberlieferung. Die Behauptung des Herausgebers (S. 24), BL sei durch Glosseme entstellt, wird durch die Thatsache, dafs die Lesart von BL zweimal am Rand von V mit dem Vermerk $\gamma \rho(\dot{\alpha} \varphi \varepsilon)$ notiert ist, nicht bewiesen, sondern widerlegt; also ist auch sonst bei der Konstitution des Textes von BL auszugehen: 3, 15. 4, 18. 22. 23. 30. 5, 7. 8. 9. 20

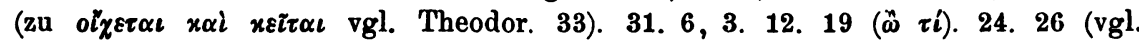
Kontost. 197). 7, 6. 8, 3. 4. 5 (s. oben S. 509). 8. 13.15 ( $\dot{\omega} s$ statt $x \alpha l$ ). 16. 20. 